\title{
Was bringt die stärkere Senkung des LDL-Cholesterins durch eine Kombinationstherapie?
}

Erland Erdmann

Um arteriosklerotische Komplikationen zu vermeiden, sollte bei Patienten mit kardiovaskulären

Erkrankungen eine Senkung des LDL-Cholesterins angestrebt werden. Zunächst wird in diesen

Fällen ein Statin eingesetzt. Doch was tun, wenn sich die empfohlenen Zielwerte allein damit

nicht erreichen lassen? Nach den Ergebnissen der IMPROVE-IT-Studie scheint Ezetimib hier

vielversprechend. Der Autor dieses Standpunkts äußert einige kritische Gedanken dazu.

Statine in der Sekundärprophylaxe I Es besteht kein Zweifel an der günstigen Wirkung der LDLCholesterin-Senkung durch Statine für die Sekundärprophylaxe kardiovaskulärer Erkrankungen. Deshalb empfehlen die Leitlinien eine Statingabe bei allen Patienten

- mit koronarer Herzkrankheit,

- nach einem Herzinfarkt,

- einer Bypass-Operation oder

- einer Katheter-interventionellen Koronarrevaskularisation.

In der Primärprävention schneiden Statine nicht so gut ab, so dass es, besonders bei Patienten mit niedrigem Risiko, durchaus kontroverse Ansichten dazu gibt [1,2]. Grundsätzlich gehen wir aber davon aus, dass zur Vermeidung arteriosklerotischer Komplikationen ein möglichst niedriges LDL angestrebt werden sollte [3].

Kombination - aber womit? | Häufig stellt sich die Frage, ob weitere Lipidsenker verordnet werden sollten, wenn sich das LDL mit Statinen alleine nicht in den von den meisten Leitlinien vorgeschlagenen Bereich (z.B. $<100 \mathrm{mg} / \mathrm{dl}$ oder bei sehr hohem Risiko $<70 \mathrm{mg} / \mathrm{dl}$ ) senken lässt. Im Augenblick bietet sich für diese Behandlung zusätzlich zu den Statinen nur noch Ezetimib an, da alle anderen zusätzlich zu Statinen eingesetzten Medikamente (z. B. Niacin, Fenofibrat, Aleglitazar, Omega-3-Fettsäuren) in kontrollierten, randomisierten Studien nur die Laborwerte verbesserten, aber weder die Zahl der kardiovaskulären Ereignisse noch die Todesrate senkten [4-8].

IMPROVE-IT | Nach den jetzt veröffentlichten Ergebnissen der über 10 Jahre laufenden IMPROVEIT-Studie scheint Ezetimib auf den ersten Blick Vorteile zu besitzen [9]: 18144 Patienten wurden etwa 10 Tage nach einem akuten Koronarereignis (ACS) entweder mit $40 \mathrm{mg}$ Simvastatin $+10 \mathrm{mg}$ Ezetimib oder mit $40 \mathrm{mg}$ Simvastatin + Plazebo behandelt. Während dieser wirklich langen Therapiezeit sank nach 7 Jahren nicht nur das LDL deutlich, sondern auch der primäre Endpunkt der Studie (Tod aus kardiovaskulärer Ursache, wesentliches Koronarereignis oder nichttödlicher Schlag- anfall) nahm signifikant um $2 \%$ ab. Viele Kollegen sehen nur oder vorzugsweise diesen zusammengesetzten Endpunkt und frohlocken jetzt über „eine evidenzbasierte Therapiestrategie“ [10].

Schaut man sich die Ergebnisse etwas genauer an, so kam es zwar zu

- weniger Herzinfarkten (13,1 statt $14,8 \%$ ) und

- weniger Schlaganfällen (4,2 statt 4,8\%), aber die Zahl der Todesfälle war identisch:

- $15,4 \%$ mit Ezetimib

- $15,3 \%$ ohne Ezitimib.

Keine Senkung der Mortalität | Wenn die Zahl der Todesfälle nach dieser langen Behandlungszeit durch Ezetimib zusätzlich zu Statinen trotz LDLSenkung nicht reduziert wurde, obwohl weniger Herzinfarkte und weniger Schlaganfälle auftraten, dann kann etwas nicht stimmen. Auch heutzutage sterben trotz unserer intensiven Bemühungen mehr Patienten im Gefolge eines Herzinfarktes oder Schlaganfalls als ohne diese Ereignisse. Wenn die Reduktion der Mortalität trotz Abnahme der wesentlichen kardiovaskulären Zwischenfälle (Herzinfarkt und Schlaganfall) noch nicht einmal im Trend zu erkennen ist, muss man logischerweise folgern, dass Ezetimib die Letalität aus anderer Ursache etwas erhöht hat.

Beispiel ASS I Man fragt sich, ob es für diese Annahme Beispiele gibt. Tatsächlich senkt ASS in den meisten Studien zur Primärprophylaxe zwar die Zahl der Herzinfarkte, nicht aber die Mortalität, da die günstigen Wirkungen durch Blutungskomplikationen aufgehoben werden [11]. Diese Tatsache wird übrigens für ASS auch in einer neuen kontrollierten, randomisierten Untersuchung an 14464 Patienten (60-85 Jahre) mit Hypertonie, Dyslipidämie und Diabetes mellitus bestätigt (Japanese Primary Prevention Project) [12]: Es gab zwar signifikant weniger Herzinfarkte unter $100 \mathrm{mg}$ ASS täglich (0,30 statt 0,58\%) und weniger transitorische ischämische Attacken (0,26 statt $0,49 \%)$, dafür aber mehr Blutungen 
$(0,86$ statt $0,51 \%)$. Infolgedessen war die Gesamtmortalität in beiden Gruppen nach 5 Jahren absolut gleich [12].

Weitere Beispiele I Es ist gar nicht so außergewöhnlich, dass Surrogatparameter durch Medikamente verbessert werden, das eigentliche Therapieziel - längeres Überleben - aber verfehlt wird. Man denke zum Beispiel an die Antiarrhythmika nach Herzinfarkt, HDL-steigernde Substanzen (u.a. Torcetrapib) oder Homocystein-senkende Vitamin-B-Kombinationspräparate. Gerade bei einer prophylaktischen Therapie müssen auch sehr seltene Nebenwirkungen beachtet werden, die sich im langen Behandlungsverlauf eben doch negativ auswirken.

Effekt niedrigerer LDL-Zielwerte | Seit langem wissen wir, dass eine Erhöhung der Statindosis das LDL weiter senkt, aber keineswegs immer eine Reduktion der Mortalität zur Folge hat. Diese Reduktion der Mortalität schien lediglich in einer Studie beim ACS zu gelingen [13].

- In der PROVE-IT-TIMI-22-Studie bei initial stationären Patienten mit ACS sank das LDL deutlich von $95 \mathrm{mg} / \mathrm{dl}$ unter $60 \mathrm{mg}$ Pravastatin auf $62 \mathrm{mg} / \mathrm{dl}$ unter $80 \mathrm{mg}$ Atorvastatin; die Gesamtmortalität hatte sich nach 2 Jahren von 3,2\% auf $2,2 \%$ verringert [13].

- Hingegen wurde in 3 weiteren kontrollierten Großstudien kein auch noch so kleiner Unterschied in der Gesamtmortalität zwischen $80 \mathrm{mg}$ Atorvastatin und $20 \mathrm{mg}$ Simvastatin oder $10 \mathrm{mg}$ Atorvastatin gefunden, obwohl die LDL-Werte unter der höheren Statindosis deutlich absanken [14-16].

Nun könnte man meinen, die Untersuchungszeiten seien auch mit einem mittleren Followup von 5-7 Jahren zu kurz gewesen. Es gibt aber keinen Beweis dafür, dass ein z.B. 10-jähriges Follow-up ein anderes Resultat gezeigt hätte. Es ist schon erstaunlich, dass die kardiovaskuläre Risikoreduktion in diesen Studien ([14-16], mit Ausnahme von [13]) nur durch Generierung eines zusammengesetzten Endpunktes signifikant erschien - bei absolut unveränderter Mortalität! Verfechter der Hochdosistherapie werden mehr Wert auf die eine positive Studie legen, während kritische Therapeuten alle Studien berücksichtigt haben wollen und zumindest auf die wissenschaftliche Unsicherheit hinweisen.

Bessere Lebensqualität? | Es leuchtet mir überhaupt nicht ein, ein keineswegs nebenwirkungsfreies Medikament zur kardiovaskulären Prophylaxe zu verordnen, das auch nach langer Therapiezeit von 10 Jahren weder das Befinden verbessert noch die Mortalität im Vergleich zu Plazebo reduziert. Nun könnte man einwenden, dass die damit behandelten Patienten zwar nicht länger leben, dafür aber besser oder leistungsfähiger. Auch dafür gibt es keine beweisenden Fakten.

\section{Konsequenz für Klinik und Praxis}

- Wenn das Ziel der prophylaktischen Therapie - ein besseres oder längeres Leben - nicht erreicht wird, sondern sich lediglich Surrogatparameter (Laborwerte, Einzelfaktoren) verändern, dann ist diese Behandlung nicht generell zu empfehlen.

- Das gilt meines Erachtens für ASS in der Primärprophylaxe genauso wie für Ezetimib in der Kombination mit Statinen.

\section{Literatur}

1 Taylor F, Huffman MD, Macedo AF et al. Statins for the primary prevention of cardiovascular disease. Cochrane Database Syst Rev 2013; CD004816

2 Abramson JD, Rosenberg HG, Jewell N, Wright JM. Should people at low risk of cardiovascular disease take a statin? BMJ 2013; 347: f6123

3 Stone N, Robinson J, Lichtenstein A et al. 2013 ACC / AHA Guideline on the Treatment of Blood Cholesterol to Reduce Atherosclerotic Cardiovascular Risk in Adults. Circulation 2014; 129 (Suppl 2): S1-S45

4 The AIM-HIGH Investigators. Niacin in patients with low $\mathrm{HDL}$ cholesterol levels receiving intensive statin therapy. N Engl J Med 2011; 365: 2255-2267

5 The HPS2-THRIVE Collaborative Group. Effects of extended-release niacin with laropiprant in highrisk patients. N Engl J Med 2014; 371: 203-212

6 The ACCORD Study Group. Effects of combination lipid therapy in type 2 diabetes mellitus. N Engl J Med 2010; 362: 1563-1574

7 Erdmann E, Califf R, Gerstein H et al. Effects of the dual peroxisome proliferator-activated receptor activator aleglitazar in patients with Type 2 Diabetes mellitus or prediabetes. Am Heart ] 2015; 170: 117-122

8 The Risk and Prevention Study Collaborative Group. $n-3$ Fatty acids in patients with multiple cardiovascular risk factors. N Engl J Med 2013; 368: 1800-1808

9 Cannon CH, Blazing M, Giugliano R et al. Ezetimibe added to statin therapy after acute coronary syndromes. N Engl J Med 2015; 372: 2387-2397

10 Custodis F, Laufs U. LDL-Cholesterin: Von der Hypothese zur Kausalität. Dtsch Med Wochenschr 2015; 140: 761-764

11 Antithrombotic Trialists Collaboration, Aspirin in the primary and secondary prevention of vascular disease. Lancet 2009; 373: 1849-1860

12 Ikeda Y, Shimada K, Teramoto T et al. Low-dose aspirin for primary prevention of cardiovascular events in Japanese patients 60 years or older with atherosclerotic risk factors. JAMA 2014; 312: 2510-2520

13 Cannon CH, Braunwald E, McCabe $C$ et al. Intensive versus moderate lipid lowering with statins after acute coronary syndromes. N Engl J Med 2004; 350: 1495-1504

14 Pedersen T, Faergeman O, Kastelein et al. High-dose atorvastatin vs usual-dose simvastatin for secondary prevention after myocardial infarction. JAMA 2005; 294: 2437-2445

15 SEARCH Collaborative Group. Intensive lowering of $\mathrm{LDL}$ cholesterol with $80 \mathrm{mg}$ versus $20 \mathrm{mg}$ simvastatin daily in 12064 survivors of myocardial infarction: a double-blind randomised trial. Lancet 2010; 376: 1658-1669

16 LaRosa J, Grundy S, Waters D et al. Intensive lipid lowering with atorvastatin in patients with stable coronary disease. N Engl J Med 2005; 352: 1425-1435

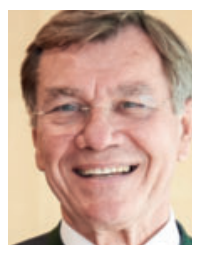

Prof. Dr. med. Erland Erdmann

ist em. Direktor der Klinik III für Innere Medizin, Herzzentrum der Universität zu Köln erland.erdmann@ uni-koeln.de

Interessenkonflikt

Der Autor gibt an, dass keine Interessenkonflikte bestehen.

DOI 10.1055/s-0041-103886 Dtsch Med Wochenschr 2015; 140: 1314-1315 (c) Georg Thieme Verlag KG . Stuttgart - New York . ISSN 0012-0472 